\title{
Entanglement Explained by Sequencing Satisfies Lack of Trajectory and The Holographic Principle
}

\author{
Bhekuzulu Khumalo \\ 305 - 19 Northern Heights, RichmondHill, Ontario, Canada \\ Bhekuzulu.khumalo@gmail.com
}

\begin{abstract}
The implications of entanglement have scared many, but if we use all we understand about existence sequencing is probably the answer given probable experiments that we should have the technology to do within the next 5 years. The simpler the easier to understand. This paper is in the tradition of thought experiments, not all have access for modern technology, a thought of the mind, given that we are further introducing algorithms into not only physics but life in general, sequencing is merely an algorithm.
\end{abstract}

Indexing terms/Keywords: algorithm of information, algorithms, entanglement, holographic principle, sequencing, trajectory

Subject Classification: optics, information theory

Type (Method/Approach): Quasi-Experimental; logic

Language: English

Date of Publication: $30-06-2018$

DOI: $10.24297 / j a p . v 14 i 2.7472$

ISSN: 2347-3487

Volume: 14 Issue: 2

Journal: Journal of Advances in Physics

Website: https://cirworld.com

This work is licensed under a Creative Commons Attribution 4.0 International License. 


\section{Introduction}

Entanglement has brought about a tremendous amount of debate and awe, is it possible to transform information faster than light. It is information being transmitted, everything is information, that we do not yet fully comprehend this information that is being transmitted and therefore categorize it, we are informed that this force is there otherwise entanglement will not be possible. Everything is information, and knowledge is just information known to us. If there is a god, he/ she is information, they basically will inform us what they are.

Physics is about experimentation, real experiments and proposed experiments because they can't be done or too expensive for the author. Alain Aspect began the current debate about entanglement with his experiment in 1982 whereby he seemingly proved that even when moving in opposite directions at or near the speed of light a signal can be sent from one particle to another seemingly instantaneously.

\section{Significance:}

The significance of this paper is to introduce the concept of sequencing with proper experiments that should be possible in the near future, very different from example publishing about multiverses, but something that can be proved or disproved in the near future.

\section{Towards Sequencing:}

Take figure 1, it shows a particle, particle, it takes time $\mathrm{t} 1$ for this particle to travel its body length. This is an illustration, particles are not balls but it will make one understand what is being proposed and it can be backed by experimentation most importantly.

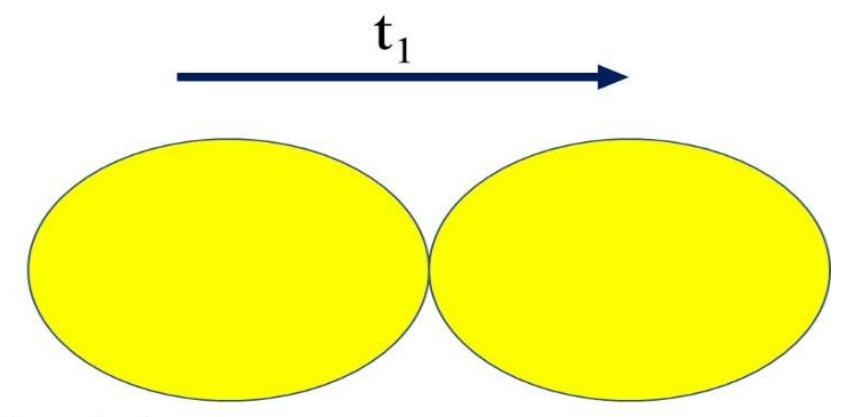

\section{Particle A}

\section{Figure 1}

Figure 1: Particle moving one body length

It takes time $t_{1}$ for particle $A$ to move one body length, this value we can get from experimentation, as nothing can go faster than light we will understand this to be the most basic practical unit of time possible, this will be understood immediately when we go to figure 2. It is at this basic unit of time that we can compare entanglement and the speed of light.

In figure 2, we take figure 1 and add two more particles, $B$ and $C . B$ and $C$ are entangled, given the reality that nothing can move faster than light, that is our foundation, $A$ change in $B$ will take time t 1 to affect a change in $B$, equal to the time it takes particle $A$ to move a body length. 


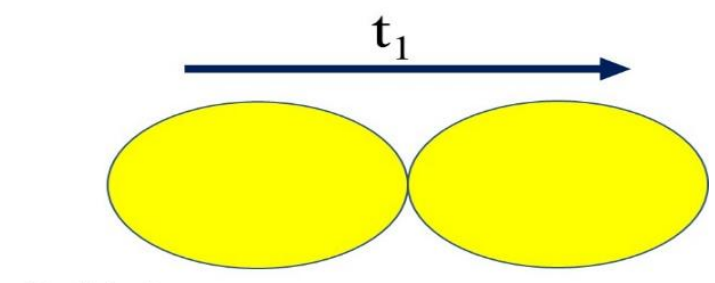

Particle A

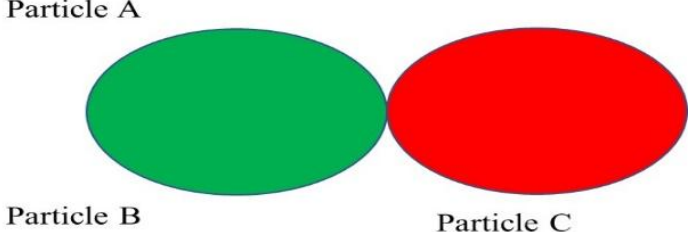

\section{Figure 2}

Figure 2: Speed of Light and Entanglement

This change in $B$ and resultant change in $C$ is defined by entanglement, but most importantly we understand why the smallest practical unit of time as defined as a body length, anything smaller is meaningless, it is at this basic unit of time that we can compare entanglement to actual movement of a particle. Figure 2 accepts those who reject Aspects experiments entanglement can not go faster than light, only at equal speed. At least one day this can be experimented, and it is not in the distant future.

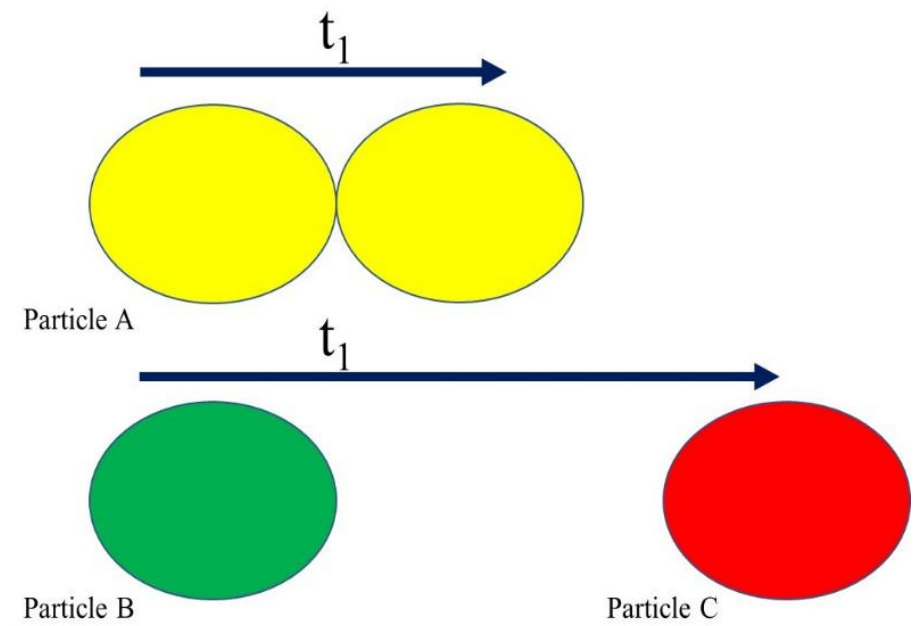

\section{Figure 3}

Figure 3: Conclusion of Aspec's Experiment

Figure 3 illustrates the controversy around Aspect's experiment and those that have copied them ever since. They believe they have the scientific evidence to support figure 3 , whereby particle $C$ has been moved away from $B$, any distance but it will still take time $t 1$ for particle $C$ to react to a change in particle $B$. This is the sticky point, but need it be so sticky. We need to return to figure 1 and allow the particle to move further than just one body length, figure 4 allows the particle to move two body lengths. 


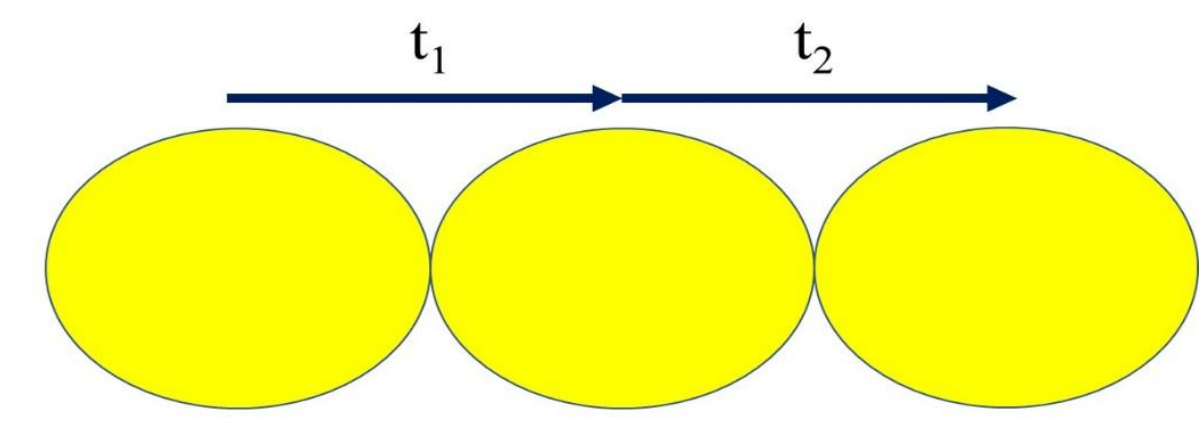

Particle A

\section{Figure 4}

Figure 4: Particle Moving Two Body Lengths

Figure 4 illustrates a particle moving two body lengths and it takes time $t_{2}$ to achieve this. Figure 5 is similar to figure 2 but another particle is added, particle $D$. Particle $D$ is entangled with particle $C$ but not with particle $B$. and particle $C$ is entangled with particle $B$. Thus a change in particle $B$ will not affect particle $D$ directly otherwise the thought experiment will fail.

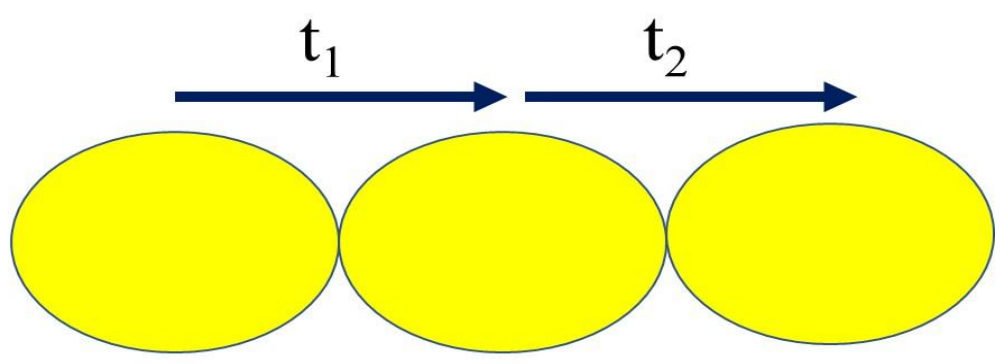

Particle A

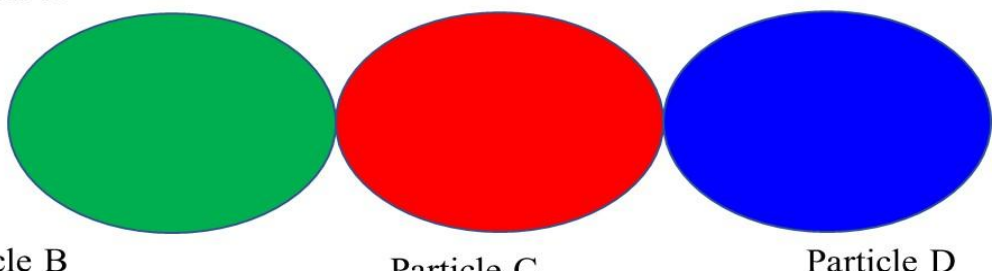

Particle B

Particle C

Particle D

\section{Figure 5}

Figure 5: Speed of Light and Entanglement II

Given that nothing can move faster than light, thus a change in B will take $t_{2}$ for a reaction in D. Given the scenario of figure 5 , it satisfies the conditions that nothing can move faster than light. To understand why some people believe that entanglement is seemingly faster than light we need two more illustrations.

Figure 6 like figure 3 illustrates the controversy around Aspect's experiments, it is a controversy otherwise Aspect would have won a Nobel Prize, it is as simple as that. Figure 6 says that no matter how far apart, it will take time $t_{2}$ for $D$ to react to changes in $B$, even if $D$ is on the other side of the universe, suggesting something that light can not compete with. 


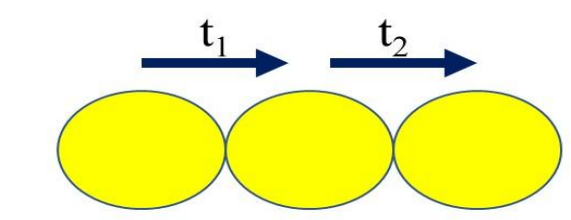

Particle A

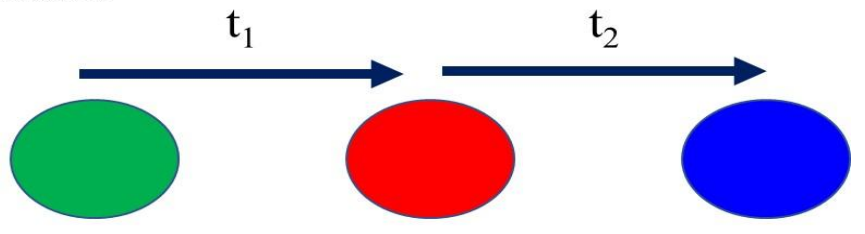

Particle B

Particle C

Particle D

\section{Figure 6}

Figure 6: Controversy of Aspects Experiment

Perhaps idea of light and entanglement competing is what confuses us, it has still taken time $t_{2}$ for $D$ to react. But even if $D$ is on other side of universe, has anything really travelled faster than the speed of light, or is it an illusion, and this illusion is answered by the thought experiment illustrated in figure 7.

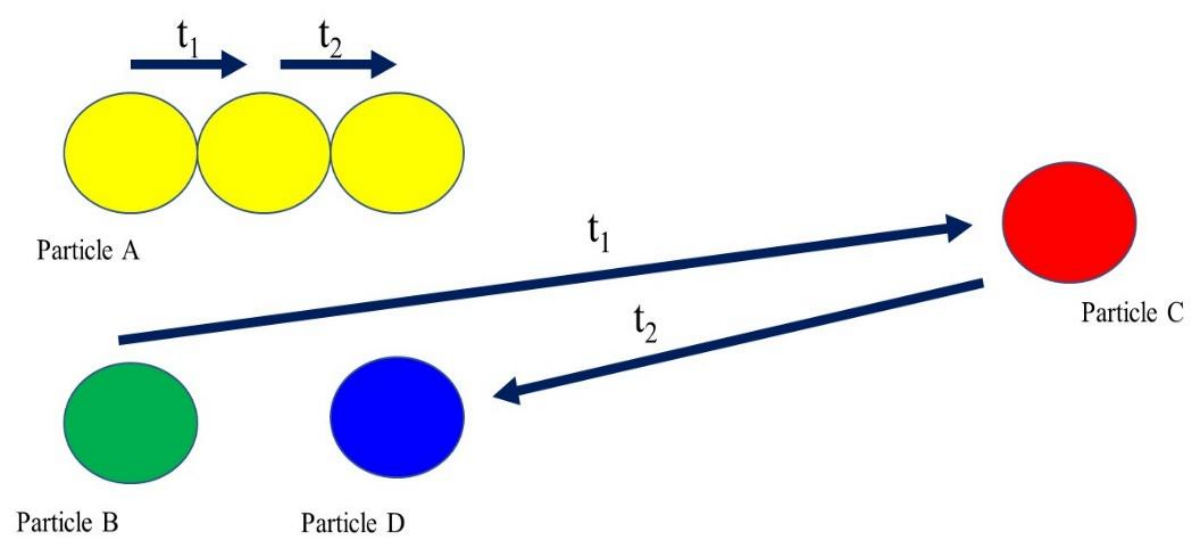

\section{Figure 7}

Figure 7: Thoughts of Sequencing

Figure 7 illustrates the principle of sequencing in the universe, that there is an order to how things be, order in terms of action, 2 can not come before 1 for instance in the number line, then it is not the second. This is all that figure 7 illustrates. Particle $D$ is 2 particle lengths from particle $B$, thus it should take time $t 2$ to react to particle $B$, thus satisfying those who say that nothing can move faster than light, satisfying Einstein. However, particle $C$ is far away thus satisfying Aspect's supporters. Note, $C$ must react before $D$, and the idea on instantaneous has fallen to accommodate those believing in Einstein's view, but the body length of a particle might as well be instantaneous, all where wrong who talked of instantaneous events and proof of breakdown of mathematics, the reaction is $t_{1}$, the smallest practical unit of time in our existence, who knows when we find out what is going on with dark matter.

Given the scenario of figure 7 , if it takes time $t_{2}$ for particle $D$ to react then at some level distance becomes an illusion and our view of time needs to change with this, but obviously only an experiment can satisfy, it's not like we are dealing with beginning of universe whereby one can say what they like. 


\section{Does Mind Experiment Support Theory:}

What theories does sequencing satisfy? In 1929, a paper was published by Neville Mott entitled ${ }^{1}$ The Wave Mechanics of $\alpha$ Ray Tracks. This paper was written one feels as a result of the fact that in cloud chambers electrons emitted leave behind tracks, straight line tracks sometimes, how does that happen when it is expected that patterns will be random. Mott made a statement that was very much ahead of its time, "The difficulty that we have in picturing how it is that a spherical wave can produce a straight track arises from our tendency to picture the wave as existing in ordinary three dimensional space, whereas we are really dealing with wave functions in the multispace formed by the co-ordinates both of the $\alpha$-particle and of every atom in the Wilson chamber." There is no trajectory as such even if it looks like there is.

This is still a puzzling issue, take the website where ideas in physics are exchanged, a question was asked ${ }^{2}$ Does a photon follow a straight trajectory in a thin transparent film? This question was asked by one who calls him/ herself Mercury. One calling themselves CuriousOne answered as such, "@Mercury: Because a photon doesn't move, at all. It's the result of a local measurement on a quantum field. The straight line approximation between any two measurements of "a photon" only exists in human minds." Mercury still could not understand and replied, "I can not understand you. Do you mean that light (photons) hasn't traveled from stars to us?".

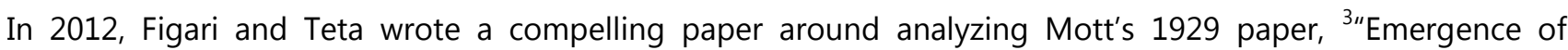
Classical Trajectories in Quantum Systems: The Cloud Chamber Problem in the Analysis of Mott(1929)". In this paper we appreciate a problem, "Darwin approaches a collision problem in the framework of Wave Mechanics with the aim to "take a problem which would be regarded at first sight as irreconcilable with a pure wave theory, but thoroughly typical of the behavior of particles, and show how in fact the correct result arises naturally from the consideration of waves alone."

He emphasizes that in order to obtain the correct predictions on the behavior of a given system $S$ One has to take into account its interaction with (part of) the environment $\varepsilon$. Therefore the wave function $\psi$ is not a wave in ordinary three dimensional space but rather it is a function of the coordinates of $S$ and of $\varepsilon . "$

When we look at figure 7, the core of the experiment being proposed to demonstrate sequencing, there is no trajectory. The experiment being proposed would satisfy the ideals of Mott as well as strongly suggest that trajectory is only because of the system, $\mathrm{S}$ interacting with the environment, $\varepsilon$, but at it's most basic system, basic particles do not adhere to our illusions of trajectory. This is the idea behind quantum decoherence, figure 7 , and entanglement in general is before quantum decoherence.

Zeh a German reader and thinker in the field in his ${ }^{4} 2005$ paper explained, "For microscopic objects, which can be sufficiently isolated, the experimental physicist has a choice between mutually exclusive ("conjugate") measurements, while macroscopic properties are decohered by their unavoidable environment in a general and specific manner. This explains their classical appearance." Figure 7 is dealing with the microscopic level, as all such pure entanglement does.

Even a successful experiment can be left to doubts about what it implies, however, theories have moved on since Mott wrote his paper in 1929. There is now some acceptance of the ${ }^{5}$ holographic principle of which Hooft has been given reasonable credit for the term. Hooft was inspired by the fact that it had been proven that all the information of a black hole seems to be projected from a 2 dimensional surface of the blackhole. In his paper, the Holographic Principle Hooft concludes "We have reached a point where, for a proper description of the particle states in the vicinity of a black hole, a two-dimensional function is required: the momentum distribution over a two-dimensional coordinate on the horizon. In addition, this function must be further reduced, since it must effectively contain not more than one $Z(2)$ variable per surface element $A_{0}$. A comparison with a holographic photograph is quickly made. In a holographic set-up, a laser beam shines on to some three-dimensional object, and the reflected light interferes with an unperturbed laser beam The interference pattern is registered on a photographic plate. In turn, after having developed the plate, we can shine a laser beam on it. An image of the three-dimensional object re-emerges... This is what we found out 
about Nature's book keeping system: the data can be written onto a surface, and the pen with which the data are written has a finite size."

Since the writing of Hooft's paper, the idea of a holographic universe has caught on. In an article published in 2017 on the University of Southampton the works of Kostas Skenderis and others who it claims in the first sentence that " ${ }^{6 "} \mathrm{~A}$ UK, Canadian and Italian study has provided what researchers believe is the first observational evidence that our universe could be a vast and complex hologram."

A holographic universe means that what we see emanates from a 2 dimensional plane. We can talk about it as information held in 1's and 0's, either on or off. To try and explain take a computer screen, what we see is 1's and 0 's, on and off so to say. When we see, say a ball move, that pattern of on and off is moving, the pixels themselves don't actually move, just the pattern, there is no trajectory as such but to the mind we see a ball moving on the screen. Difficult concept to wrap the mind around, but that just means all ${ }^{7}$ dimensions are here right now. An excellent video to watch would be entitled, "Kostas Skenderis, Our Universe as a Hologram."

Figure 7 above satisfies these ideas in experimentation form, there is no trajectory at the basic level, it is a sequence, after this comes this, it does not matter how far away this looks like to our eyes, it is next given the system parameters that have been created, that is the importance of this paper. Sequencing satisfies both lack of trajectory and a holographic universe.

\section{The Relationship:}

How do we compare the speed of light to entanglement, one would think this unnecessary, but it is a worthwhile endeavour just for the intellectual sake. It is unnecessary because we have established that it is unwise to compare the two because technically everything is moving at the speed of light, but over the years we have conceptualized the holographic universe, things we should have grasped once we understood there is no trajectory, but the knowledge was not there, only the idea of different dimensions was there. Experiments have been discussed above that will either confirm or refute this thought. However, it is important to look at the relationship more closely, perhaps it could be helpful in the future.

When talking about what we can sense, be it through our eyes, they are photon sensors, or very sophisticated instruments that sense photons at far greater depths than the human eye. When we talk of relationships, there must be a way to verify the underlying thought behind them, the underlying thought is not as obvious, and the difficulty comes up with a thought experiment to verify the speculation.

What is the speed of entanglement, Et. We must understand this concept in two ways, the speed that we perceive things as if we are looking at them and as such the mind interprets things that way. We see a 100 meter sprinter run what we consider superfast, or we see a cheetah moving superfast, or even a jet fighter plane, and that is how we interpret how fast something is, when we talk of speed of light we think in terms of distance per second and we say wow when we consider the fastest human on earth or even the fastest rocket we have conceived. When we consider speed in such a manner then the speed of entanglement compared to light is:

$E t=c X \# P_{b}$ or $C^{2} P_{b}$ equation 1 where

$E t=$ speed of entanglement

$C=$ speed of light

$\# P_{b}=$ number of potential photons between .

This is how we perceive things as, what is fastest from point A to B. However, at its most fundamental levels nature does not behave as we see things. The double slit experiment should have warned us about this. The reality is when it comes to entanglement the known particles are entangled, there is no movement we can 
detect though obviously there is a force at work. In reality there is a sequencing phenomenon that is taking place.

$$
\begin{aligned}
& E r=S_{i \rightarrow i+1} D \quad \text { equation } 2 \text { where } \\
& E r=\text { Entanglement reality } \\
& S_{i \rightarrow i+1}=\text { next sequence } \\
& D=\text { is the function for density of information }
\end{aligned}
$$

The next sequence is what is supposed to be next. This was thoroughly explained above in explanations of figure 1 through to figure 7. ${ }^{9}$ Density of information was explained in the paper Information theory and Dimensions, density is the amount of information a body has as a whole and per cubic millimeter. The denser information is, the greater the chances of interacting with information thus slowing the process down. This can be understood when we think of what we consider normal light, it slows down denser information like glass water than it is in the air, and it is at its fastest in a vacuum where information is at its least dense. We thus give a density of information in a vacuum a value of 1 .

What does density of information mean given a scenario of no trajectory and sequencing. Density of information is about occupied potential. Note the use of occupied potential not space. A lead statue that is an exact replica of an adult might weigh half a ton, but the human only weighs 170 pounds. They both seemingly occupy the same amount of space, but the information density of that space occupied, the potential fulfilled of that space is very different, a gamma photon will be blocked by the lead replica whilst it will go through the human being. Another way to look at potential is to pick up a piece of rock and look up into the night sky and understand that rock can potentially occupy any space in this universe where there is room. The two ways of looking at potential are the same, meaning they are talking of the same concept.

When talking about density of information, what we consider as a vacuum it has been confirmed is full of matter, dark matter and dark energy. It is doubtful if dark matter will show uniformity, most likely some sort of variation. Thus, the concept of $D$ will have to be recalibrated in the near future where the value of 1 for $D$ is finer than a vacuum.

It is this variable $D$ that gives entanglement an advantage over a light beam, the density of information, does it affect entanglement in the same manner as it affects a moving photon, a ray of light. For this we need an experiment. The experiment is laid out below.

\section{Lead Block}

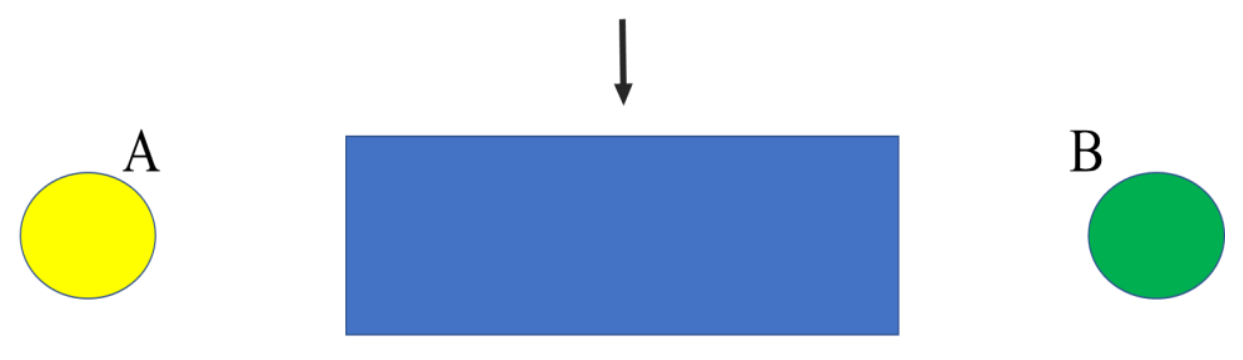

Figure 8

Figure 8 tests for density, does density of information affect entanglement as much as it affects a ray of light. We know the light that our eyes normally sense can not pass through a lead block in normal circumstances, it 
should not affect entanglement but however to keep naysayers silent an experiment has been designed. Will putting a lead block between two entangled particles affect entanglement? it is still seemingly faster than light? Has it slowed down? We are using a lead block to illustrate this applies to all photons no matter the differences in what we know as energy.

The keen observer will of course have a question to be answered, what about heat? Place enough heat energy at one end of a lead block and being a metal and thus what is considered highly heat conductive, sooner or later will begin being radiated on the other side. Depending on one's view of what heat is, an unexplainable phenomenon that we call heat radiation, or a particle known as a photon known as a ${ }^{10}$ tshison, if heat radiation is a photon, then a photon has penetrated. But let us look at the reality, for a very long while the amount of heat put in is not equal from end to end. It takes time for that to happen because the energy must be sufficient enough to allow the electromagnetic effect to allow the release of this heat radiation in equal amounts all around the lead block, a concept covered by entropy. But this effect is slow, only when the same amount of heat is being radiated from every surface of the lead block can we claim that the heat radiation is being released at equal amounts, a far cry from entanglement with a lead block in the way, if successful it is instantaneous. But what this heat radiation does tell us is the increasing extent of quantum tunneling due to increasing levels of energy.

Note equation 2 has done away with any concept of distance, replacing distance with what should follow next in the sequence and distance as such has nothing to do with that reality. This gets rid of one of the most important continuous variables, the concept of distance, there is no continuous at the basic level of information, everything is discrete. Distance is continuous because you can always hypothetically cut that distance in half.

\section{The question in entanglement moves away from what distance is covered per period to how many sequences can be made per period.}

\section{Conclusion:}

Only an experiment can satisfy, but if successful in proving figure 7, then our view of universe must change, and further evidence of no trajectory is inserted into our thinking. Remember that when you create entanglement and move a particle away from another it means it is occupying a location that could otherwise be occupied by something else. The technology that comes from lack of trajectory at this basic level of existence are yet to be comprehended but they should be mind blowing. Nutshell in her video, ${ }^{11}$ "Uncertainty Principle \& Trajectories - From Cloud Chamber Traces to Decoherence Theory" though not clearing the mind totally of doubt is a good starting point to learn about the lack of trajectory, that is idea of science, use every tool available.

What ever it is that allows entanglement to occur is information, with further investigation it will inform us more of its nature. Being information what ever is being transmitted will follow the first law of information, every relationship represents a loss of freedom, and being information it's behaviour is determined by the ${ }^{12}$ algorithm of information, and thus greatly influenced by probability.

This paper answers in part ways a question asked by Einstein in1935 as elaborated in the video ${ }^{13}$ "Quantum Entanglement Documentary - Atomic Physics and Reality" a question interpreted by John Bell, "Bohr, I think quit early in life had decided that ordinary concepts like space and time simply wouldn't work on the atomic scale. It was always unclear what Bohr would replace those concepts by if anything, but Einstein was very attached to the space-time description and thought that one should try very hard to extend it into the atomic domain and this was the root of the disagreement between the two men." Sequencing takes away the concept of distance, in terms of operation space is greatly reduced to as little as zero. Though unclear what Bohr would replace these concepts with, what ever it is must have something to do with sequencing. 


\section{References}

[1] Mott, N.F (1929) The Wave Mechanics of $\alpha$ - Ray Tracks, http://rspa.royalsocietypublishing.org/content/royprsa/126/800/79.full.pdf

[2] Mercury (2016) Does a photon follow a straight trajectory in a thin transparent film? https://physics.stackexchange.com/questions/252710/does-a-photon-follow-a-straight-trajectory-in-a-thintransparent-film

[3] Figari, R. Teta, A (2012). Emergence of Classical Trajectories in Quantum Systems: The Cloud Chamber Problem in the Analysis of Mott(1929) https://arxiv.org/abs/1209.2665

[4] Zeh, H.D (2005). Roots and Fruits of Decoherence http://www.bourbaphy.fr/zeh.pdf

[5] Hooft, G. (2000). The Holographic Principle https://arxiv.org/abs/hep-th/0003004

[6] University of Southampton, (2017) Study reveals substantial evidence of holographic universe https://www.southampton.ac.uk/news/2017/01/holographic-universe.page

[7] Khumalo, B (2015) Dimensions and Mysterious of the universe, http://instituteofknowledge.blogspot.ca/2015/04/dimensions-and-mysterious-of-universe.html

[8] Southampton Education School, (2013) Kostas Skenderis, Our Universe as a Hologram, https://www.youtube.com/watch?v=XoPOlzBC9mo

[9] Khumalo, B (2015) Information theory and dimensions: Enhancing Quantum Mechanics. Journal of Advances in Physics https://cirworld.com/index.php/jap/article/view/1352\#.WrXzHpqrpK8.facebook

[10] Khumalo, B (2015) Heat, quantum mechanics and information . Journal of Advances in Physics. https://cirworld.com/index.php/jap/article/view/1328

[11] Cracking the Nutshell, (2017), https://www.youtube.com/watch?v=m1t7sRMZqQU

[12] Khumalo, B. (2017) Knowledge Economics: Summary and Rationality. Modern Economy, 8, 1533-1560. https://file.scirp.org/Html/10-7201720_81147.htm

[13] Ray, M (2014) Quantum Entanglement Documentary - Atomic Physics and Reality. https://www.youtube.com/watch?v=BFvJOZ51tmc 